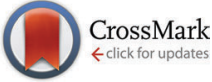

Cite this: Phys. Chem. Chem. Phys., 2017, 19, 2304

Received 16th May 2016,

Accepted 5th December 2016

DOI: $10.1039 / \mathrm{c} 6 \mathrm{cp} 03328 \mathrm{~h}$

www.rsc.org/pccp

\title{
Interfacial water on organic substrates at cryogenic temperatures: hydrogen bonding and quantification in the submonolayer regime
}

\author{
D. Houdoux, J. Houplin, L. Amiaud, A. Lafosse* and C. Dablemont
}

\begin{abstract}
Water molecules were used to probe the physical and chemical properties of a model hydrophilic organic organized layer. To this end, $\mathrm{H}_{2} \mathrm{O}$ adsorption on mercaptoundecanoic acid self-assembled monolayers (SAMs) was investigated at the molecular level under ultra-high vacuum by high resolution electron energy loss spectroscopy (HREELS), through the sensitivity of the water $\mathrm{OH}$ stretching modes to the molecular environment. The water interfacial layer formation and structure were studied upon deposition at $28 \mathrm{~K}$. A direct sensitive quantification in the submonolayer regime (10-80\% of completion) was achieved by the sole measurement of the $\mathrm{OH}$ stretching mode frequencies, and the dominant basic $\left(-\mathrm{COO}^{-}\right)$/acidic $(-\mathrm{COOH})$ forms of the terminal functions could be probed. The surface densities of the water interfacial layer and the SAM terminal functions were measured independently, and demonstrated to be comparable. This means that the SAM terminal functions provided anchors for water adsorption through two hydrogen bonds and that the SAM acted as a template even at $28 \mathrm{~K}$. Upon annealing at $110 \mathrm{~K}$, the water molecules were observed to form clusters of higher molecular density, dewetting the supporting substrate. Finally, the vanishing of the supporting substrate vibrational signature, due to the masking effect by the deposited water layer, was used to estimate the depth probed by HREELS through water layers to be $11 \pm 2 \AA$.
\end{abstract}

\section{Introduction}

Water vapour interaction with surfaces is of general technological interest for preserving or even for manipulating interfacial physical and chemical properties. Dissociative or non-dissociative water adsorption was intensively investigated at the molecular level for metallic ${ }^{1-5}$ and metal oxide substrates. ${ }^{1,2,6-8}$ Another important type of interfaces, although less considered, are organic layers. ${ }^{1,9-12}$ The study of the interaction between vapourphase probe molecules and supported molecular platforms participates in the fundamental understanding of molecular recognition-based chemical sensors, double-layer structures and biological membrane interfaces. ${ }^{13-15}$ The main types of molecular interactions driving adsorption on organic interfaces are: electrostatic (ion-ion) binding interactions, covalent bonding, hydrogen bonding and acid-base interactions. ${ }^{13,16-18}$

Self-assembled monolayers (SAMs) are model organic layers, since they are organized surface-confined monolayers of functionalized compounds. They provide structured molecular platforms of controlled chemical composition for developing chemical and biological sensors. ${ }^{15,19}$ In particular, layers having acidic

Institut des Sciences Moléculaires d'Orsay (ISMO), CNRS, Univ. Paris Sud, Université Paris Saclay, F-91405 Orsay, France. E-mail: anne.lafosse@u-psud.fr terminal functions provide chemical anchors suitable for peptide and protein immobilization. ${ }^{16,17,20,21}$ Therefore, it is of prime interest to study the interaction of such platforms with their surrounding media, and in particular with the ubiquitous compound water. ${ }^{9}$ As an example, SAMs of 11-mercaptoundecanoic acid (MUA, HS- $\left.\left(\mathrm{CH}_{2}\right)_{10}-\mathrm{COOH}\right)$ are used as model hydrophilic acid terminated alkanethiolate systems providing 2D-ordered layers of terminal functions. The latter are able to act as water anchors through hydrogen bond(s). The specific behaviour of interfacial water $^{22}$ will be taken advantage of to probe some physical and chemical properties of the prepared molecular platforms.

In this paper, in order to study the water-SAM interfacial layer at the molecular level, the $\mathrm{H}_{2} \mathrm{O}$ adsorption has been studied at its very first stages under ultra-high vacuum (UHV) by high resolution electron energy loss spectroscopy (HREELS), a vibrational spectroscopy technique known for its high surface sensitivity. Isothermal adsorption measurements have been performed at $28 \mathrm{~K}$ on base $\left(-\mathrm{COO}^{-}\right)$and acid $(-\mathrm{COOH})$ terminated MUA substrates, by exposing them to controlled $\mathrm{H}_{2} \mathrm{O}$ doses under background deposition conditions. At this temperature, the water sticking and condensation coefficients are equal to unity. ${ }^{23}$ In this system, the water-SAM interfacial layer structure results from a delicate interplay between different hydrogen bonding interactions: the $-\mathrm{COOH}$ terminal functions are 
embedded into an intralayer hydrogen bond network, water molecules are hydrogen bonded to the acidic/basic terminal functions, and the water molecules also form a hydrogen bond network when close enough. ${ }^{11,12,22,24}$ The $\mathrm{OH}$ stretching modes of water are known to be sensitive to its molecular environment and the $\nu(\mathrm{OH})^{\mathrm{H}_{2} \mathrm{O}}$ vibrations are red-shifting upon the formation of hydrogen bonds. ${ }^{1,3,4,6-8}$ Therefore, special attention was given to the loss feature extending from 380 to $470 \mathrm{meV}$.

In the submonolayer regime (0-1 L), a direct quantification of the interfacial water layer completion was achieved, based on the sole peak position of the hydrogen bonded $\mathrm{OH}$ stretching band. At the same time, molecular surface packing densities were measured quantitatively for the water-layer under formation as well as for the supporting MUA molecular platforms. The molecular density of the water-MUA interfacial layer has been shown to be low enough to match the MUA terminal function density. In the multilayer regime $(2-16 \mathrm{~L})$, the formation of porous amorphous solid water (ASW) layers was observed, as expected from the chosen experimental conditions. ${ }^{23,25,26}$ The vanishing of the MUA substrate vibrational signature, due to the masking effect, ${ }^{27}$ has been used to estimate the depth probed by HREELS through the deposited water layers to be $11 \pm 2 \AA$.

The water-MUA interfacial layer structuration is driven by competing hydrogen bonding interactions: anchoring to the substrate terminal functions, and water-water bonding. Therefore, water molecules were used to probe the dominant basic/acidic forms $\left(-\mathrm{COO}^{-} /-\mathrm{COOH}\right)$ of the carboxylic acid terminal functions. The $\mathrm{H}_{2} \mathrm{O}$-terminal function interactions were observed to be strong enough for the underlying MUA organized layer to serve as a template for water molecules anchoring at $28 \mathrm{~K}$. Furthermore, when the temperature was raised to $110 \mathrm{~K}$, the mobility of the water molecules was then high enough for longrange diffusion to take place, which leads to the formation of high density water domains and "dewetting" of the SAM.

\section{Experimental}

\section{SAMs preparation}

The 11-mercaptoundecanoic acid (MUA) SAM preparation at room temperature was described in detail in a previous paper. ${ }^{28}$ Briefly, MUA was purchased from Sigma-Aldrich (98\%, SaintQuentin Fallavier, France). All solvents were of reagent grade. Reagents were used without any further purification. Glass substrates $(11 \mathrm{~mm} \times 11 \mathrm{~mm})$ coated successively with a $2.5 \mathrm{~nm}$ thick layer of chromium and a $250 \mathrm{~nm}$ thick layer of gold were purchased from Arrandee (Werther, Germany). The gold substrates were annealed by a brief passage in a flame, and then cleaned by 30 minutes of UV-ozone treatment. Then, they were immersed in a freshly prepared $10 \mathrm{mM}$ solution of thiol in absolute ethanol for 2 hours. After thorough rinsing in ethanol (15 minutes), the surfaces were rinsed in aqueous solution for 15 minutes. During the last rinsing step, it was possible to control, to a certain extent, the surface composition by using an acidic ( $\mathrm{pH}$ 2) or a basic ( $\mathrm{pH} 9)$ solution. ${ }^{29}$ Since the $\mathrm{p} K_{\mathrm{a}}$ of the acidic function of MUA is around $4.5-5$ in solution, ${ }^{30}$ the terminal carboxylic functions of the MUA SAMs were mainly in their acidic $(-\mathrm{COOH})$ or basic $\left(-\mathrm{COO}^{-}\right)$form. However, a complete acidic or basic surface can never be obtained because of the existence of an acid-base equilibrium. ${ }^{16,21}$ After the acidic or basic rinsing step, the surfaces were dried under a flow of dry nitrogen.

\section{Water deposition}

The SAMs were loaded into the UHV system (base pressure below $2 \times 10^{-10}$ Torr) through a load-lock chamber, allowing the samples to be transferred into UHV, without baking the vacuum chambers. The SAM samples were then fixed at the end of a He closed-cycle cryostat and cooled down to $\sim 28 \mathrm{~K}$. Microleak valves allowed depositing fractions of water on the samples. The water amount was controlled by regulating the introduced partial pressure (typically $10^{-9}$ Torr or even $10^{-8}$ Torr for the higher deposits) and the exposure time. During the experiments, the pressure value was recorded every 10 seconds starting from and ending at $3.8 \times 10^{-10}$ Torr, threshold pressure for notable variations, and below which the partial pressure of the residual gas is no more negligible. For each exposure, the read pressure was integrated over time to determine precisely the adsorbed amount of water $\theta$ in Langmuir (L). The sticking and condensation coefficients for water adsorption are equal to 1 at $\sim 28 \mathrm{~K}^{23,25,31}$

Absolute measurements of low pressures $\left(<10^{-9}\right.$ Torr $)$ in a vacuum chamber are very delicate and depend on many parameters: ${ }^{32-34}$ temperature, residual gas conductance, nonuniformity of pumping inside the vacuum chamber and gauge history. The read pressure values were corrected by a gauge calibration factor. The uncertainty on the water exposure was estimated to be around $15 \%$ because of (i) the uncertainty in the reading of the gauge and its calibration factor and (ii) the additional contribution of the background pressure (UHV system residual vacuum, residual water in the chamber and in the gas inlet).

\section{HREEL characterization of SAMs}

The pristine and water exposed MUA SAMs were characterized by HREEL spectroscopy at $\sim 28 \mathrm{~K}$. The spectrometer consists of a double monochromator and a single analyzer (model IB 500 by Omicron). The energy loss spectra $\left(-15 \leq E_{\text {loss }} \leq 500 \mathrm{meV}\right)$ were recorded for the incident energy $E_{\mathrm{i}}=6 \mathrm{eV}$, with an overall resolution $\Delta E_{\mathrm{FWHM}} \sim 6-7 \mathrm{meV}$, measured at the full width at half maximum (FWHM) of the elastic peak. All the spectra were measured in the specular geometry $\left(\theta_{\mathrm{i}}=\theta_{\mathrm{f}}=55^{\circ}\right.$ with respect to the surface normal) and are presented without any normalization. The electron induced damaging of the films during the long accumulation times was regularly checked for.

\section{Results and discussion}

\section{HREELS characterization of acidic/basic MUA SAMs and ASW multilayers}

1.1. Characteristic vibrational signature of MUA SAMs. The curve in Fig. 1a and the bottom black curve in Fig. 1b display the energy loss spectra recorded at $28 \mathrm{~K}$ for basic $\left(-\mathrm{COO}^{-}\right)$and 


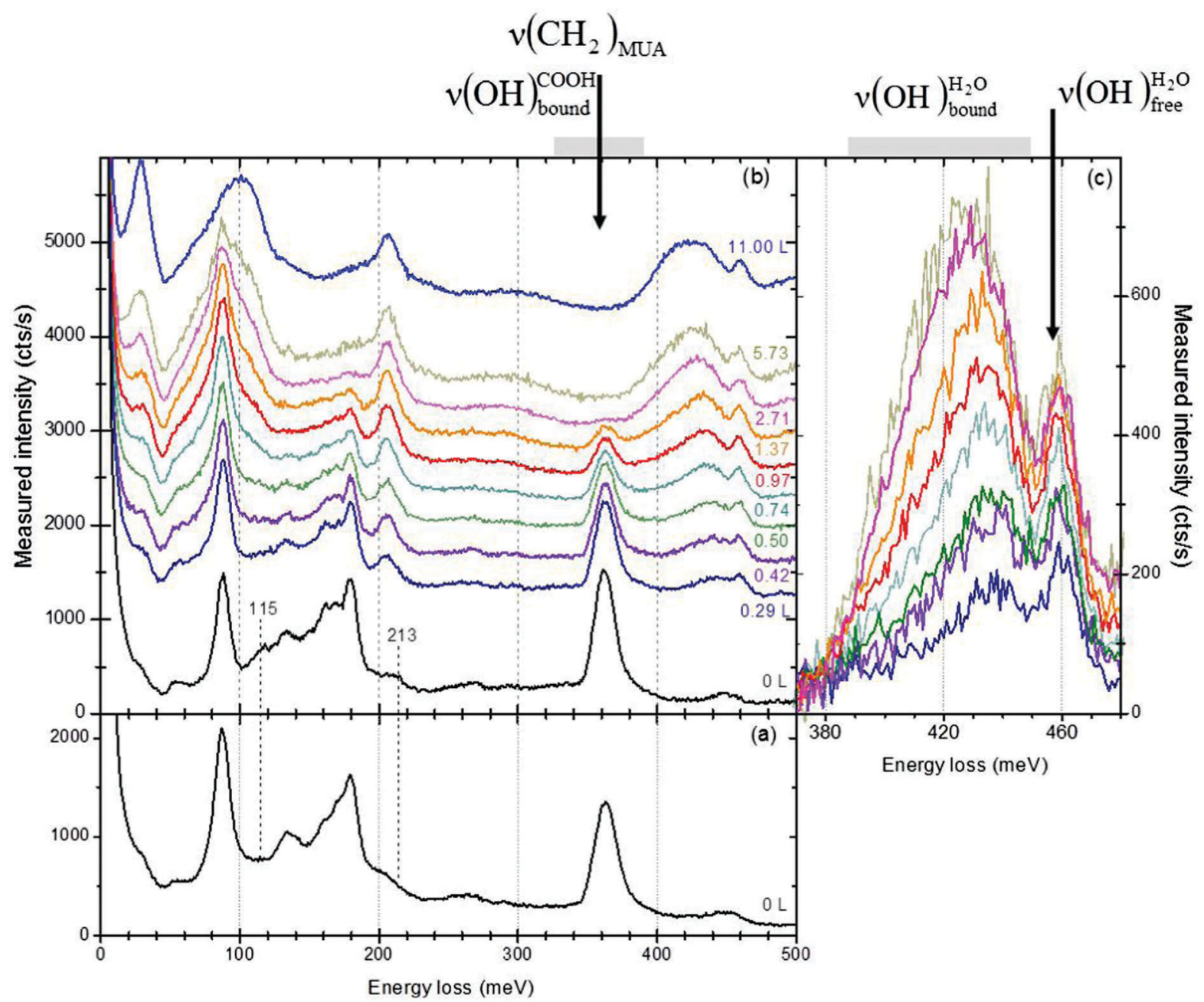

Fig. 1 Energy loss spectra $\left(E_{\mathrm{i}}=6 \mathrm{eV}, \Delta E_{\mathrm{FWHM}} \sim 7 \mathrm{meV}\right.$ ) of a pristine basic (panel (a)), acidic MUA SAM before (bottom black curve) and after successive water exposures $\theta=0.29 \mathrm{~L}, 0.42 \mathrm{~L}, 0.50 \mathrm{~L}, 0.74 \mathrm{~L}, 0.97 \mathrm{~L}, 1.37 \mathrm{~L}, 2.71 \mathrm{~L}, 5.73 \mathrm{~L}, 11.00 \mathrm{~L}$ (panel (b)). The values of the doses are cumulated ones and the spectra are vertically shifted for clarity reasons. The $\nu(\mathrm{OH})^{\mathrm{H}_{2} \mathrm{O}}$ bands are compared (without a vertical shift) in panel (c).

acidic (-COOH) MUA SAMs, respectively. For the latter one, the attributions of the main resolved structures were previously discussed. ${ }^{28,35}$ The energy loss spectrum of the basic MUA SAM resembles the acidic one. Note that the two broad signatures between 250 and $268 \mathrm{meV}$ and around $450 \mathrm{meV}$ are attributed to multiple losses (e.g. ref. 36 and 37). They result from twofold $88 \mathrm{meV}+[162-180 \mathrm{meV}]$ and $88 \mathrm{meV}+362 \mathrm{meV}$ inelastic scattering processes, respectively. The main differences between acidic and basic SAMs are, as expected, related to the terminal function: (i) the $\nu(\mathrm{C}=\mathrm{O})$ stretching mode observed at $213 \mathrm{meV}$ for the acidic SAM shifts down to $198 \mathrm{meV}$ for the basic SAM, (ii) the out-of-plane bending of two hydrogen bonded terminal functions at $115 \mathrm{meV}$ decreases in intensity (as a consequence of the equilibrium existing between the acidic and the basic forms). The most obvious difference that should be expected between the acidic and basic forms should be related to the vibrational signature of the $\mathrm{OH}$ bond, although characterized by weak oscillating forces. ${ }^{38}$ On one hand, the $\nu(\mathrm{OH})_{\text {free }}^{\mathrm{COOH}}$ vibration mode is expected at $\sim 444 \mathrm{meV}^{35,39-43}$ in a region where strong multiple losses are contributing. On the other hand, the proximity of molecules as well as their flexibility allow the formation of hydrogen bonds between neighbouring $\mathrm{COOH}$ groups, ${ }^{24,29,44}$ broadening and shifting the $\mathrm{OH}$ stretching $\nu(\mathrm{OH})_{\mathrm{bound}}^{\mathrm{COOH}}$ down to $325-390 \mathrm{meV}^{28}{ }^{28}$ Therefore, $\nu(\mathrm{OH})_{\text {free }}^{\mathrm{COOH}}$ and $\nu(\mathrm{OH})_{\text {bound }}^{\mathrm{COOH}}$ cannot be isolated in the spectrum.

1.2. Characteristic vibrational signature of ASW multilayers. Amorphous solid water (ASW) multilayers deposited on an MUA SAM (top blue curve on Fig. $1 \mathrm{~b}-\theta \approx 11 \mathrm{~L}$ ) display the same energy loss spectra as ASW multilayers deposited on hydrogenated diamond ${ }^{39}$ due to the limited HREELS probed depth. In particular, the broad peak $(380-459 \mathrm{meV})$ is ascribed to the OH stretching modes $\nu(\mathrm{OH})_{\text {bound }}^{\mathrm{H}_{2} \mathrm{O}}$ and the sharp peak at $459 \mathrm{meV}$ to the unbound (i.e. without interactions) $\nu(\mathrm{OH})_{\text {free }}^{\mathrm{H}_{2} \mathrm{O}}$.

\section{From a water-SAM interfacial layer to ASW multilayers}

2.1. Depth probed by HREELS. Fig. $1 \mathrm{~b}$ displays the energy loss spectra of an acidic MUA SAM after several water deposits. The deposited quantities are cumulated ones, resulting from successive exposures all performed one after the other on the 
sample maintained at $28 \mathrm{~K}$. It allows reducing sample manipulation, reaching appropriate deposit amounts in a single day but induces cumulative uncertainties in the deposited amount. As the probed depth of the electrons in HREELS is limited, for low deposits, both the substrate and deposited water are probed, but for higher deposits, only water is probed. Consequently, above $\theta \gtrsim 2.8 \mathrm{~L}$ water layers, the substrate is no more visible. The question of the HREELS probed depth in organic materials, as our MUA SAM, is still open. ${ }^{45}$ The extinction of the loss at $362 \mathrm{meV}$, attributed to the methylene stretching modes $\nu\left(\mathrm{CH}_{2}\right)_{\text {MUA }}$, determines the limit above which the MUA SAM is no longer probed. Such a method has been proposed for Langmuir-Blodgett PMMA (poly(methyl methacrylate)) sandwich structures. ${ }^{27}$ In our case, at $6 \mathrm{eV}$, the $\nu\left(\mathrm{CH}_{2}\right)_{\text {MUA }}$ signal of the underneath MUA SAM drops below the detection limit for $\theta \gtrsim 2.8 \mathrm{~L}$ water layers. The thickness of water monolayers deposited on inorganic and SAM substrates has been estimated to be $2.82 \AA$ (ref. 6) and $3.66 \AA$ (ref. 10), respectively. Taking into account the presence of the $\mathrm{COOH}$ terminal function in between the water layer and the SAM methylene groups, the total effective probed depth is estimated to be $11 \pm 2 \AA$ for the $\nu\left(\mathrm{CH}_{2}\right)_{\text {MUA }}$ vibrational stretch band at an electron energy of $6 \mathrm{eV}$.

2.2. $\nu(\mathrm{OH})_{\text {bound }}^{\mathrm{H}_{2} \mathrm{O}}$ red-shifting at increasing water exposure. As the deposited water amount increases (Fig. 1c), the sharp peak $\nu(\mathrm{OH})_{\text {free }}^{\mathrm{H}_{2} \mathrm{O}}$ remains at $459 \mathrm{meV}$, whereas there is a displacement of the broad peak $\nu(\mathrm{OH})_{\text {bound }}^{\mathrm{H}_{2} \mathrm{O}}$ to lower energy losses, as expected. ${ }^{4,7}$ This displacement will be used to quantify the amount of deposited water thanks to a precise positioning of that peak.

To take into account only the water layer signature, the recorded spectra were corrected from the MUA SAM contribution, as long as the characteristic $\nu\left(\mathrm{CH}_{2}\right)_{\text {MUA }}$ signature remained visible (for $\theta \lesssim 2.8 \mathrm{~L}$ ). Doing so, the small contribution of the multiple loss $88+362 \mathrm{meV}$ was removed from the $\nu(\mathrm{OH})_{\text {bound }}^{\mathrm{H}_{2} \mathrm{O}}$ feature, but not the residual contribution $\nu(\mathrm{OH})_{\mathrm{free}}^{\mathrm{COOH}}$ of any free $-\mathrm{COOH}$ terminal functions expected at $444 \mathrm{meV}$ with weak oscillating force. ${ }^{38}$ The MUA SAM background contribution was subtracted, after scaling the pristine spectrum (bottom black spectrum on Fig. $1 \mathrm{~b})$ to the $\nu\left(\mathrm{CH}_{2}\right)_{\text {MUA }}$ peak intensity. The spectrum resulting from the subtraction contains only the water layer contribution and will be called the corrected spectrum in the following section. The position of the water broad peak is determined for each deposited layer thanks to a fitting procedure, using two Gaussians (the position of one being fixed at $459 \mathrm{meV}$ and the second one left free).

The results of water deposition on a series of acidic and basic SAMs are presented in the calibration curve displayed in Fig. 2. With increasing water exposure, the $\nu(\mathrm{OH})_{\text {bound }}^{\mathrm{H}_{2} \mathrm{O}}$ peak shifts towards lower energy losses. The water $\mathrm{OH}$ bond environment is modified and the hydrogen bond network evolves towards ASW. The asymptotic value is $\nu_{0, \mathrm{ASW}}=423.6 \pm 0.2 \mathrm{meV}$, which is a signature of amorphous ice and not the crystallized one. ${ }^{3,11,46}$ A poorly organized water phase involving, for example, only 2.5 hydrogen bonds per water molecule leads to a $\nu(\mathrm{OH})^{\mathrm{H}_{2} \mathrm{O}}$ vibration at $422 \mathrm{meV}^{6}$ By contrast, a fully crystallized ice,

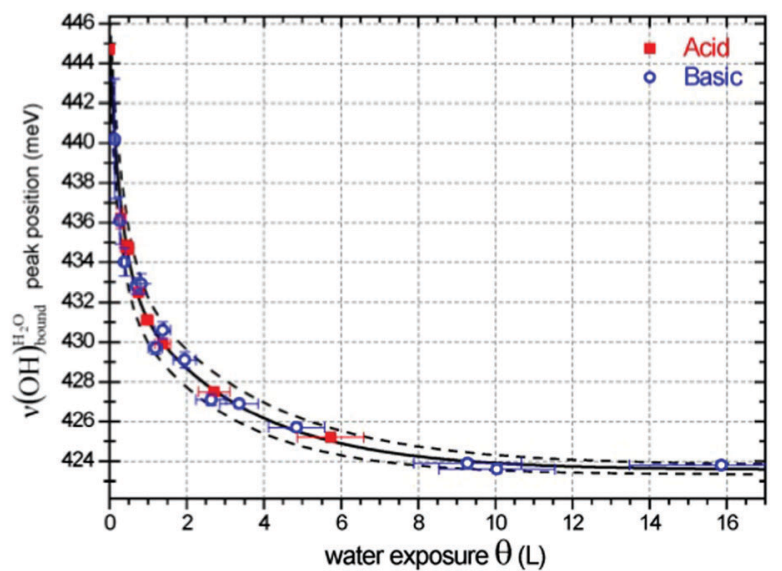

Fig. 2 Calibration curve: $\nu(\mathrm{OH})_{\text {bound }}^{\mathrm{H}_{2} \mathrm{O}}$ peak position (in meV) as a function of the water exposure $\theta$ (L) for acidic (red full squares) and basic (blue open circles) MUA SAMs. The black curve gives the fit by a sum of two exponentials describing the observed trend.

$$
\nu=A_{1} \exp \left(-\frac{\theta}{\theta_{1}}\right)+A_{2} \exp \left(-\frac{\theta}{\theta_{2}}\right)+\nu_{0}
$$

with $\theta_{1}=0.30 \pm 0.09 \mathrm{~L}, \theta_{2}=3.0 \pm 0.7 \mathrm{~L}, A_{1}=11.1 \pm 0.7 \mathrm{meV}, A_{2}=$ $10.0 \pm 0.6 \mathrm{meV}$, and $\nu_{0}=423.6 \pm 0.2 \mathrm{meV}$. The dashed curves are the limit empirical variation laws deduced from the uncertainties in the fitting parameters.

presenting 4 hydrogen bonds per water molecule, is characterized by a vibration at the $400 \mathrm{meV}$ peak. ${ }^{6}$

These experiments have been performed for both acidic and basic samples. The tendency that is observed is independent of the sample type. The curve in Fig. 2 allows the direct determination of the water amount present on the surface, in the submonolayer regime, thanks to the sole value of the $\nu(\mathrm{OH})_{\text {bound }}^{\mathrm{H}_{2} \mathrm{O}}$ vibrational peak in HREELS. This procedure can be applied to any MUA samples (acidic, basic or mixed) and for any tuning of the spectrometer.

2.3. From MUA-assisted adsorption to an ASW multilayer. In order to estimate the amount of water necessary to reach the completion of the water-SAM interfacial layer, a study based on the intensity of the vibration modes associated with the MUA SAM, $\nu\left(\mathrm{CH}_{2}\right)_{\text {MUA }}$, and the deposited water, $\nu(\mathrm{OH})^{\mathrm{H}_{2} \mathrm{O}}$, has been performed. The intensity variations of the relative areas $\Gamma$ along water exposure allow monitoring both the underlying SAM and the water layer under formation. Keeping in mind that quantitative studies based on feature integrated areas in HREELS are known to be challenging, ${ }^{45}$ HREEL spectra after deposition of each water layer have been recorded with the same settings of the spectrometer. The relative area $\Gamma_{\nu\left(\mathrm{CH}_{2}\right)}$ of the $\nu\left(\mathrm{CH}_{2}\right)_{\mathrm{MUA}}$ peak is obtained by integration between 345 and $390 \mathrm{meV}$, and normalization by the pristine SAM, representing a bare surface (100\% of free surface, only the SAM contribution in the probed depth). The underlying contribution of $\nu(\mathrm{OH})_{\text {bound }}^{\mathrm{COOH}}$ is neglected as it is of weak intensity on a very broad domain and is removed by the baseline correction. The relative area $\Gamma_{\nu(\mathrm{OH})}$ of the $\nu(\mathrm{OH})^{\mathrm{H}_{2} \mathrm{O}}$ feature is obtained by integration between 390 and $475 \mathrm{meV}$ of the corrected spectra, and by normalization with respect to the 


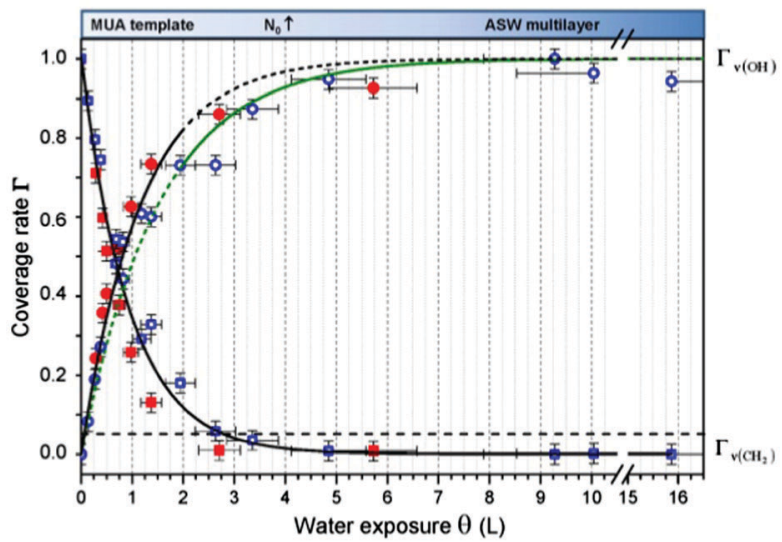

Fig. 3 Normalized area $\Gamma$ under the features attributed to $\nu(\mathrm{OH})^{\mathrm{H}_{2} \mathrm{O}}$ (circles) and $\nu\left(\mathrm{CH}_{2}\right)_{\mathrm{MUA}}$ (squares) with respect to the water exposure $\theta(\mathrm{L})$ for acidic (red full symbols) and basic (blue open symbols) MUA SAMs. The detection limit is represented by the dashed line at $\Gamma \sim 0.05$. The black curves are the exponential variation laws predicted by a Langmuir model for layer growth (eqn (1) and (2)). They were obtained by adjusting one single parameter $\theta_{\text {lim }}$ over the range $0-2 \mathrm{~L}$, i.e. during the formation of the water-SAM interfacial layer. The green curve represents the exponential law associated with the completion of the ASW multilayer, taking place over the range $2-5.5 \mathrm{~L}$.

highest deposited amount, representing a fully multilayer covered surface $\left(100 \%\right.$ of water on the surface, no more $\mathrm{CH}_{2}$ in the probed depth). It contains the contribution of $\nu(\mathrm{OH})_{\text {free }}^{\mathrm{H}_{2} \mathrm{O}}, \nu(\mathrm{OH})_{\text {bound }}^{\mathrm{H}_{2} \mathrm{O}}$ and eventually $\nu(\mathrm{OH})_{\text {free }}^{\mathrm{COOH}}$. The present study is mainly focused on the water-SAM interfacial layer (i.e. in the very low exposure regime), so that any intrinsic $\nu(\mathrm{OH})^{\mathrm{H}_{2} \mathrm{O}}$ band intensity variation can be neglected since the probed water molecules all primarily interact with the SAM terminal functions.

Fig. 3 displays the evolution of the normalized areas $\Gamma_{\nu(\mathrm{OH})}$ and $\Gamma_{\nu\left(\mathrm{CH}_{2}\right)}$ with respect to the water exposure $\theta$. As expected, when water exposure increases the $\Gamma_{\nu(\mathrm{OH})}$ increases before reaching saturation, while the $\Gamma_{\nu\left(\mathrm{CH}_{2}\right)}$ decreases. More specifically, we note that:

(i) The $\Gamma_{\nu\left(\mathrm{CH}_{2}\right)}$ signal goes below the detection limit for $\theta \gtrsim 2.8 \mathrm{~L}$ : the MUA surface is completely covered for $\theta \approx 2.8 \mathrm{~L}$.

(ii) The $\Gamma_{\nu(\mathrm{OH})}$ normalized area saturates to 1 for $\theta \gtrsim 5.5 \mathrm{~L}$ : the probed depth contains only ASW ice.

(iii) For $2.8 \lesssim \theta \lesssim 5.5 \mathrm{~L}$, the probed depth only contains water and the signal continues to increase, as a sign of increasing quantities of water molecules inside the probed depth.

2.3.1. Low exposure regime $\theta \lesssim 2.0 \mathrm{~L}$. In the low coverage regime $\theta \lesssim 2.0 \mathrm{~L}$, the normalized area $\Gamma$ experimentally determined under the $\nu(\mathrm{OH})^{\mathrm{H}_{2} \mathrm{O}}$ (respectively $\nu\left(\mathrm{CH}_{2}\right)_{\text {MUA }}$ ) peak represented in Fig. 3 can be adjusted by exponential variation laws (1) and (2) characterized by a single parameter $\theta_{\text {lim }}$.

$$
\begin{gathered}
\Gamma_{\nu(\mathrm{OH})}=1-\exp \left(-\frac{\theta}{\theta_{\lim }^{\mathrm{H}_{2} \mathrm{O}}}\right) \\
\Gamma_{\nu\left(\mathrm{CH}_{2}\right)}=\exp \left(-\frac{\theta}{\theta_{\lim }^{\mathrm{MUA}}}\right)
\end{gathered}
$$

The adjustment parameters are found to be $\theta_{\lim }^{\mathrm{H}_{2} \mathrm{O} \text {,low-deposit }}=$ $1.16 \pm 0.17 \mathrm{~L}$ and $\theta_{\mathrm{lim}}^{\mathrm{MUA}}=0.94 \pm 0.14 \mathrm{~L}$. Using a simple Langmuir model of monolayer deposition, ${ }^{47,48} \theta_{\lim }$ is proportional to the adsorption site density $N_{0}$.

$$
N_{0}=\theta_{\lim } \times K
$$

with $K=\frac{N_{\mathrm{A}}}{\sqrt{2 \pi \mathrm{MRT}}}=4.8 \times 10^{14}$ molecules $\mathrm{cm}^{-2} \mathrm{~L}^{-1}$.

$N_{\mathrm{A}}$ is Avogadro's constant, $R$ is the perfect gases constant, $T$ is the gas temperature in $\mathrm{K}$ (here approximated $300 \mathrm{~K}$ ), $M=$ $18 \times 10^{-3} \mathrm{~kg} \mathrm{~mol}^{-1}$ is the molar mass of the gaseous species. The adjusted $\theta_{\text {lim }}$ values leads to $N_{0}^{\mathrm{H}_{2} \mathrm{O} \text {,low-deposit }}=4.7-6.5 \times$ $10^{14}$ molecules $\mathrm{cm}^{-2}$ and $N_{0}^{\mathrm{MUA}}=3.8-5.2 \times 10^{14}$ molecules $\mathrm{cm}^{-2}$. According to Schreiber et al., ${ }^{49}$ a thiolated aliphatic molecule occupies a $21.6 \AA^{2}$ surface inside a SAM, which corresponds to a molecule surface density of $4.6 \times 10^{14}$ molecules $\mathrm{cm}^{-2}$. This is in a perfect agreement with the density $N_{0}^{\mathrm{MUA}}$ determined above.

$N_{0}^{\mathrm{H}_{2} \mathrm{O} \text {,low-deposit }}$ and $N_{0}^{\mathrm{MUA}}$ values are quite comparable. It means that, in the low exposure regime, the impinging entities build a layer having a density comparable to the packing density of the MUA SAM. The SAM terminal functions serve as anchors for the incoming molecules and act as a template for building the water layer even at $28 \mathrm{~K}$. Since the obtained density is far below that expected for water molecule assemblies alone, ${ }^{23,26}$ the adsorbed water molecules strongly interact with the SAM. According to the diffusion model developed by Kasza et al., ${ }^{31} \dagger$ at $28 \mathrm{~K}$ water molecules linked by one hydrogen bond are still mobile, while two hydrogen bonds immobilize them. So we conclude that the water molecules are anchored to the SAM via two hydrogen bonds, resulting in an adsorption energy estimated to be $\sim 10 \mathrm{kcal} \mathrm{mol}^{-1}$. This is in accordance with the estimation, obtained by TPD, for water adsorption at a low coverage on a 16-mercaptohexadecanoic acid HS- $\left(\mathrm{CH}_{2}\right)_{15}$-COOH SAM. ${ }^{11}$ Some studies have considered water interfacial layers with silicon oxide $^{6}$ and muscovite mica ${ }^{8}$ at room temperature. In both cases, they proposed that the structure of the first water layer is driven by the substrate, in accordance with our observation.

2.3.2. Multilayer deposition regime $\theta \gtrsim 2 L$. In the higher coverage regime, the adjustment parameter, coming from eqn (1), is $\theta_{\mathrm{lim}}^{\mathrm{ASW}}=1.52 \pm 0.26 \mathrm{~L}$. This means that, in the multilayer formation regime under our experimental conditions, the limit is amorphous porous ice as $N_{0}^{\mathrm{ASW}}=\theta_{\mathrm{lim}}^{\mathrm{ASW}} \times \mathrm{K}=7.3 \pm 1.3 \times$ $10^{14}$ molecules $\mathrm{cm}^{-2}$. From this value, an estimate of the water multilayer volumic mass can be obtained through the simple relationship

$$
\rho=\frac{N_{0, \mathrm{ASW}}^{3 / 2} \times M}{N_{\mathrm{A}}} \approx 0.59 \pm 0.16 \mathrm{~g} \mathrm{~cm}^{-3} .
$$

This is in excellent agreement with the value obtained experimentally by optical density measurements for ice deposited at $20 \mathrm{~K}^{23,50}$

$\uparrow$ The limit temperatures for diffusion were estimated to be $17 \mathrm{~K}$ and $35 \mathrm{~K}$, for water molecules linked by one and two hydrogen bond(s), respectively (taking an average diffusion activation energy of $1.25 \mathrm{kcal} \mathrm{mol}^{-1}$ for a hydrogen bond of $5 \mathrm{kcal} \mathrm{mol}^{-1}$ ). 


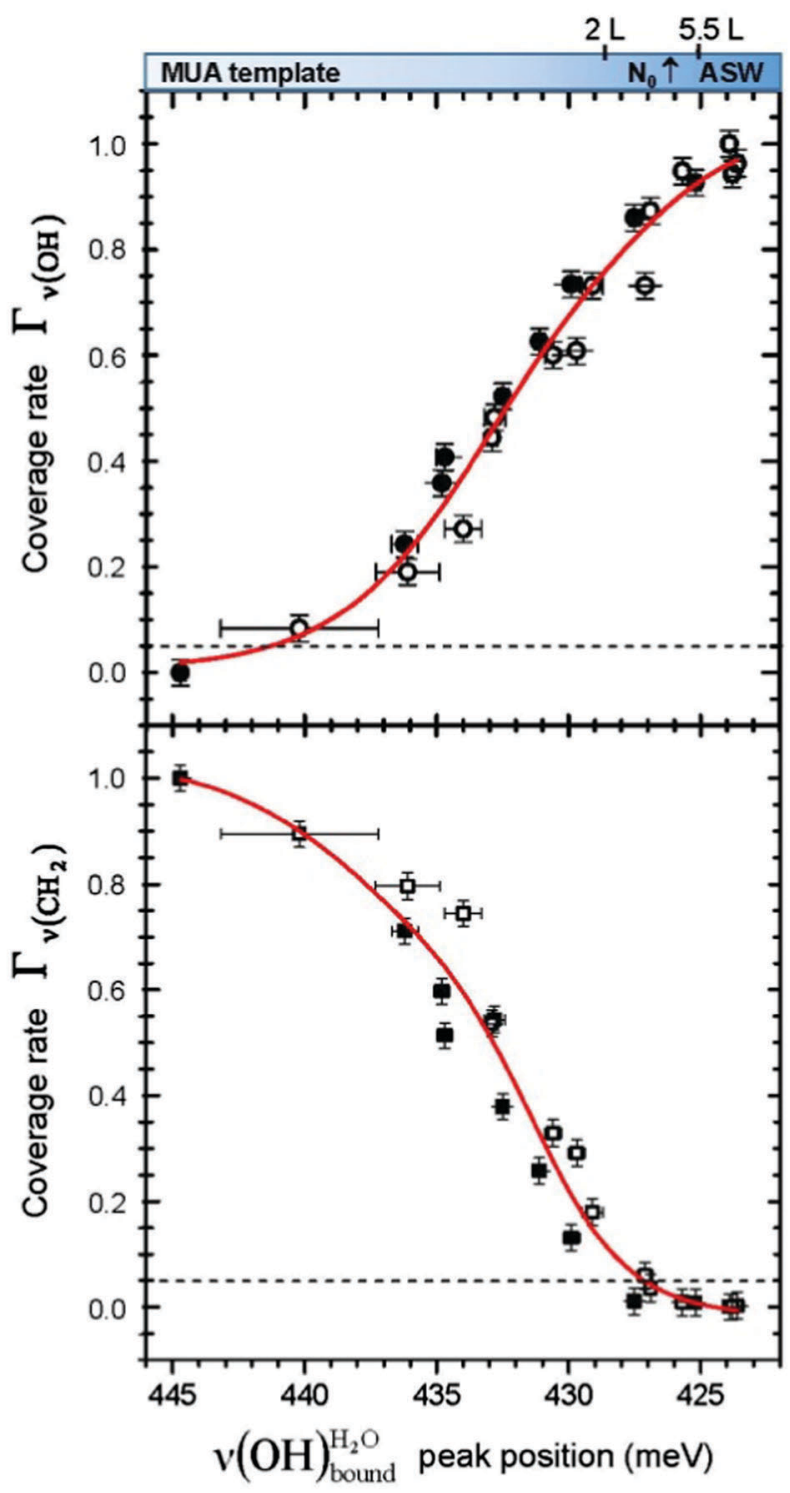

Fig. 4 Coverage rates $\Gamma_{\nu(\mathrm{OH})}$ (upper panel - circles) and $\Gamma_{\nu\left(\mathrm{CH}_{2}\right)}$ (lower panel - squares) as a function of the $\nu(\mathrm{OH})_{\text {bound }}^{\mathrm{H}_{2} \mathrm{O}}$ peak position (in meV) for acidic (full symbols) and basic (open symbols) MUA SAMs. The detection limit is represented by the dashed line at $\Gamma \sim 0.05$. The sigmoidal curves are drawn to guide the eye.

2.3.3. Direct determination of water coverage in the submonolayer regime. By combining all the experimental data (Fig. 2 and 3), the obtained peak position of the $\nu(\mathrm{OH})_{\text {bound }}^{\mathrm{H}_{2} \mathrm{O}}$ can be directly related to the coverage rates $\Gamma$ of the SAM platform (Fig. 4). For example, on a sample on which the deposited water amount is unknown, if the position of the water peak is measured at $436 \mathrm{meV}$, it can be deduced that $70-78 \%$ of the MUA molecule sites (and, so, of $\mathrm{COOH}$ ) are free and that the coverage level is $20-26 \%$. To put it in a nutshell, HREELS is sensitive to the formation of the water interfacial layer, which is on the way to its completion. In the $\mathbf{9 0 - 1 0 \% ~ d o m a i n ~ f o r ~ t h e ~ n o r m a l i z e d ~ a r e a ~}$ $\Gamma_{\nu\left(\mathrm{CH}_{2}\right)}$, the peak position for the $\nu(\mathrm{OH})_{\text {bound }}^{\mathrm{H}_{2} \mathrm{O}}$ shifts from 440 to $428 \mathrm{meV}$. This low energy loss limit corresponds to $\sim 2.4 \mathrm{~L}$ exposure and $\Gamma_{\nu(\mathrm{OH})}$ is $\sim 80 \%$, confirming that this is the beginning of the multilayer regime. In the domain of the interfacial layer completion, Fig. 4 constitutes an abacus, which is independent of the HREELS settings. It gives a direct determination of the coverage at exposures as low as submonolayer ones. In addition, the morphology of the first water layer is driven by the SAM as shown by the similarity of the surface density of water for the low deposits and the surface density of the MUA SAM. As demonstrated above, a water molecule explores the surface before immobilization on one terminal function through two hydrogen bonds. Consequently, the arrangement of the $\mathrm{COOH} /$ $\mathrm{COO}^{-}$terminal functions of the SAM may influence the structure of the first water layer as discussed below.

\section{Water as a probe for determining the acidic/basic character of the MUA SAM}

As there is an acid-base equilibrium between $\mathrm{COOH}$ and $\mathrm{COO}^{-}$ terminal functions, the acidic or basic character of the SAM interface deserves to be probed. The energy loss spectra of the bare acidic and basic MUA SAMs resemble each other with the exceptions of the position of the weak intensity $\nu(\mathrm{C}=\mathrm{O})$ stretching mode and the intensity of the out-of-plane bending of two hydrogen bonded terminal functions. When these two types of SAMs are exposed to water, the positions of the losses are also similar (Fig. 1 - Section 1.1). However, it has been shown that each water molecule diffuses until it forms two hydrogen bonds with one terminal function.

In Fig. 5, the features attributed to $\nu(\mathrm{OH})^{\mathrm{H}_{2} \mathrm{O}}$ are compared for acid- and base-terminated MUA SAMs at low water exposures. For the higher doses $(\sim 0.7$ and $1.4 \mathrm{~L})$, they are comparable. Only, for the lowest exposures $(\sim 0.3$ and $0.4 \mathrm{~L})$, the height of the $\nu(\mathrm{OH})_{\text {free }}^{\mathrm{H}_{2} \mathrm{O}}$ peak is always smaller than the one of the $\nu(\mathrm{OH})_{\text {bound }}^{\mathrm{H}_{2} \mathrm{O}}$ on a basic SAM, whereas it is always higher on an acidic SAM. This can serve as a qualitative probe for determining the acidic/basic state of the MUA SAM interface layer. The $\nu(\mathrm{OH})_{\text {free }}^{\mathrm{H}_{2} \mathrm{O}}$ intensity reflects the proportion of terminal functions in their acidic-form - $\mathrm{COOH}$, at disposal for hydrogen bonding with incoming water. As mentioned above (Section 2.3.1),

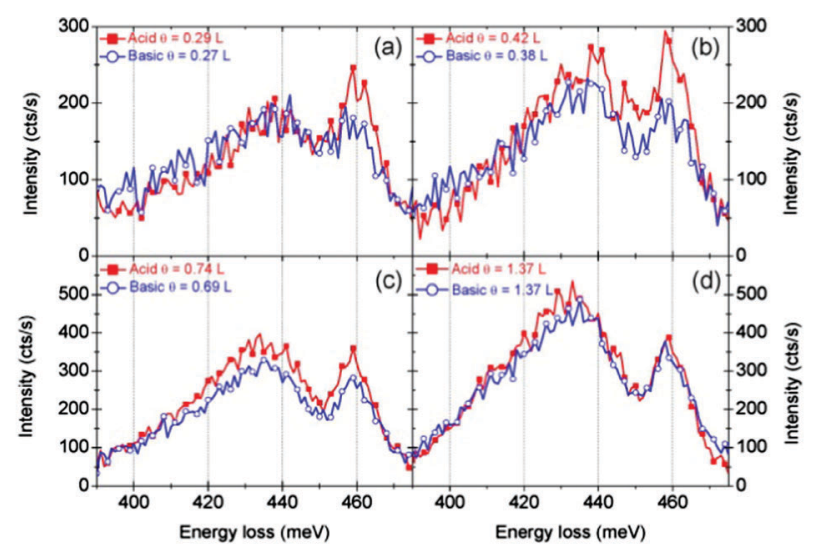

Fig. 5 Comparison of the $\nu(\mathrm{OH})^{\mathrm{H}_{2} \mathrm{O}}$ bands for acid (red full squares) and base (blue open circles) terminated MUA SAMs at low water exposures: (a) $\theta \sim 0.3 \mathrm{~L}$, (b) $\theta \sim 0.4 \mathrm{~L}$, (c) $\theta \sim 0.7 \mathrm{~L}$, and (d) $\theta \sim 1.4 \mathrm{~L}$. 
water molecules bind to the SAM terminal functions through two hydrogen bonds. When interacting with an acidic-form - $\mathrm{COOH}$ terminal function, a water molecule might involve only one of its covalent $\mathrm{OH}$ bonds. As the acidic SAM is both a hydrogen bond donor and acceptor, the second hydrogen bond might come from the covalent $\mathrm{OH}$ bond of the carboxylic acid function. On the contrary, basic SAMs are only hydrogen bond acceptors as they have no covalent $\mathrm{OH}$ bond to involve into intermolecular interactions with water. So, water molecules create bonds using their two $\mathrm{OH}$ covalent bonds to interact with basic SAMs, leading to a reduced $\nu(\mathrm{OH})_{\text {free }}^{\mathrm{H}_{2} \mathrm{O}}$ intensity.

\section{Favouring water/water and $\mathrm{COOH} / \mathrm{COOH}$ interactions through annealing}

The influence of water deposition on an acidic MUA SAM itself is now examined thanks to the as-recorded spectrum of a water layer of $0.50 \mathrm{~L}$ deposited at $28 \mathrm{~K}$ (green curve of Fig. 6). The vibrational signatures, that are characteristic of a water interfacial layer, are observed with a broad peak between 380 and $450 \mathrm{meV}$ attributed to $\nu(\mathrm{OH})_{\text {bound }}^{\mathrm{H}_{2} \mathrm{O}}$ and a peak at $459 \mathrm{meV}\left(\nu(\mathrm{OH})_{\text {free }}^{\mathrm{H}_{2} \mathrm{O}}\right)$. However, there is an additional signature at $443 \mathrm{meV}$, which can be attributed to $\nu(\mathrm{OH})_{\text {free }}^{\mathrm{COOH}}$, meaning that the intra-SAM hydrogen bond network was broken..$^{35,39-43}$ The associated loss of energy is counterbalanced by the formation of hydrogen bonds between adsorbing water molecules and the SAM. Statistically, a $0.50 \mathrm{~L}$ deposit is favourable for the observation of this phenomenon. The probability to have two free $\mathrm{COOH}$ functions close enough to each other to regenerate the hydrogen bond network observed in the pristine MUA SAM is quite low.

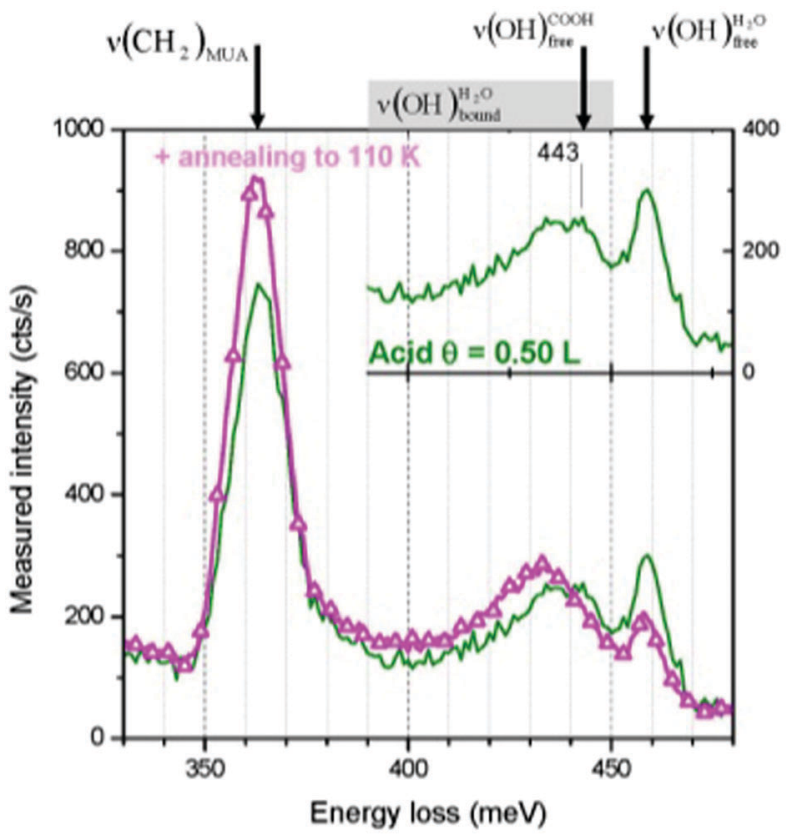

Fig. $6 \nu\left(\mathrm{CH}_{2}\right)$ and $\nu(\mathrm{OH})$ stretching regions $(330-480 \mathrm{meV}$, as recorded) of an acidic MUA SAM after $0.50 \mathrm{~L}$ water exposure at $28 \mathrm{~K}$ (green full curve, also shown in the inset) and subsequent annealing to $110 \mathrm{~K}$ (pink triangles) $\left(E_{\mathrm{i}}=6 \mathrm{eV}, \Delta E_{\mathrm{FWHM}} \sim 6 \mathrm{meV}\right)$.
After the deposition of $0.50 \mathrm{~L}$ at $28 \mathrm{~K}$, the sample is heated at $110 \mathrm{~K}$ (pink curve with triangles in Fig. 6), which is below the temperature desorption of water $(\geq 130 \mathrm{~K}) .{ }^{12,51,52}$ The area under the $\nu(\mathrm{OH})$ broad peak only slightly decreases $(12 \% \pm 5 \%)$, while the $\nu(\mathrm{OH})_{\text {free }}^{\mathrm{H}_{2} \mathrm{O}}$ peak looses half of its intensity. Consequently, a $\nu(\mathrm{OH})_{\text {free }}^{\mathrm{H}_{2} \mathrm{O}}$ conversion into $\nu(\mathrm{OH})_{\text {bound }}^{\mathrm{H}_{2} \mathrm{O}}$ is observed. The $\nu(\mathrm{OH})_{\text {bound }}^{\mathrm{H}_{2} \mathrm{O}}$ peak is shifted towards the lower energy losses. Referring to the calibration curve in Fig. 2, it means that the initial $0.50 \mathrm{~L}$ amount of water displays, after heating, a hydrogen bond network comparable to the one of a higher deposit. Also it can be noted that the peak attributed to the $\nu(\mathrm{OH})_{\text {free }}^{\mathrm{COOH}}$ vibration disappears. This is in agreement with the rearrangement of the whole system, the water submonolayer as well as the terminal functions of the supporting SAM. At $110 \mathrm{~K}$, two hydrogen bonds are not enough to prevent water diffusion (see Section 2.3.1). ${ }^{31}$ The carboxylic acid functions no longer serve as a template for the water submonolayer. The molecules can migrate to build an optimized water network above the SAM. A possible interpretation is that the water submonolayer has now contracted into $2 \mathrm{D}$ dense islands, so that their signature mimics the one of a higher deposit. However, this $\nu(\mathrm{OH})_{\text {bound }}^{\mathrm{H}_{2} \mathrm{O}}$ displacement has to be carefully considered, since the annealing of an ice multilayer would result in comparable experimental evidence.

A stronger evidence of water contraction into $2 \mathrm{D}$ islands upon $110 \mathrm{~K}$ heating is given by the variation of the $\nu\left(\mathrm{CH}_{2}\right)$ intensity. The normalized area $\Gamma_{\nu\left(\mathrm{CH}_{2}\right)}$ increases by $\sim 0.14-0.16$, meaning that the proportion of the MUA surface free from water increases. Thereby, the water molecule surface density $N_{0}^{2 \mathrm{D}, 110 \mathrm{~K}}$ is multiplied by a factor $\sim 1.6-1.9$. By extrapolation, a hypothetical volumic mass can be estimated in the range 0.7-1.1 $\mathrm{g} \mathrm{cm}^{-3}$ from the $2 \mathrm{D}$ surface density. Such a water contraction leading to the restoring of domains with available SAM terminal functions was also observed by Engquist in the case of 2 A water layer deposited on a methyl 16-mercaptohexadecanoate $\mathrm{HS}-\left(\mathrm{CH}_{2}\right)_{15}-\mathrm{COO}-\mathrm{CH}_{3}$ SAM on gold. ${ }^{10}$ The annealing from 100 to $140 \mathrm{~K}$ decreases the fraction of water capped $\mathrm{C}=\mathrm{O}$ groups from $95 \%$ to $36 \%$. Considering that the contraction is the variation of the covered surface over the initial water coverage, a contraction of $(95-36) / 95=0.62$ was obtained. In our case, on an acidic SAM, the obtained contraction $\sim 0.46-0.54$ is in a reasonable agreement, taking into account that we are working at a much lower coverage well before the start of the multilayer. Such a metastable behaviour of water layers was observed, upon annealing up to $140 \mathrm{~K}$, for films deposited at $80 \mathrm{~K}$ on a 16-mercaptohexadecanoic acid HS- $\left(\mathrm{CH}_{2}\right)_{15}$-COOH SAM. ${ }^{11}$

\section{Conclusions}

In this paper, mercaptoundecanoic acid (MUA, HS- $\left.\left(\mathrm{CH}_{2}\right)_{10}-\mathrm{COOH}\right)$ SAMs were chosen as model hydrophilic organized surfaceconfined organic layers to study water interaction and adsorption at the molecular level. $\mathrm{H}_{2} \mathrm{O}$ molecule adsorption was investigated under ultra-high vacuum on both acid $(-\mathrm{COOH})$ and base $\left(-\mathrm{COO}^{-}\right)$ terminated SAMs. The sensitivity of the water $\mathrm{OH}$ stretching modes to the molecular environment was probed by high 
resolution electron energy loss spectroscopy (HREELS). Two water characteristic losses are particularly observed: one related to free $\mathrm{OH}$ bonds $\nu(\mathrm{OH})_{\text {free }}^{\mathrm{H}_{2} \mathrm{O}}$ at $459 \mathrm{meV}$ and one attributed to $\mathrm{OH}$ bonds involved in hydrogen bonding interaction $(\mathrm{s}) \nu(\mathrm{OH})_{\text {bound }}^{\mathrm{H}_{2} \mathrm{O}}$. At the very first stages of water adsorption, the latter was observed to shift from $440 \mathrm{meV}$ down to $428 \mathrm{meV}$.

Working at a low water exposure, the formation of the water interfacial layer was followed by considering both the increase in intensity of the $\mathrm{OH}$ stretching feature and the decrease in intensity of the MUA substrate vibrational signature $\nu\left(\mathrm{CH}_{2}\right)$. With the help of a simple Langmuir model, the surface densities of the water interfacial layer and the SAM terminal functions were measured independently, and demonstrated to be comparable. The water layer consists of $(5.6 \pm 0.9) \times$ $10^{14}$ molecules $\mathrm{cm}^{-2}$, which corresponds to a remarkably low extrapolated density of $0.4 \pm 0.1 \mathrm{~g} \mathrm{~cm}^{-3}$. Hence, the SAM terminal functions provide anchors for water adsorption through two hydrogen bonds and the SAM acts as a template for the interfacial water layer formation even at $28 \mathrm{~K}$. A direct quantification of the amount of deposited water was achieved in the submonolayer regime (10-80\% of completion) by the sole measurement of the $\mathrm{OH}$ stretching mode frequencies, as a consequence of the HREELS surface sensitivity. The dominant basic $\left(-\mathrm{COO}^{-}\right)$/acidic $(-\mathrm{COOH})$ forms of the terminal functions could be probed by considering the relative intensities of the $\nu(\mathrm{OH})_{\text {bound }}^{\mathrm{H}_{2} \mathrm{O}}$ and $\nu(\mathrm{OH})_{\text {free }}^{\mathrm{H}_{2} \mathrm{O}}$ loss features. Upon annealing at $110 \mathrm{~K}$, the deposited water molecules were observed to migrate and form clusters of higher molecular density, reaching values characteristic for phases where water-water interactions dominate. This contraction was accompanied by the dewetting of the supporting substrate.

At a higher water exposure at $28 \mathrm{~K}$, the progressive transition to porous ASW ice multilayers was observed as expected and led to characteristic energy losses $\nu(\mathrm{OH})_{\text {free }}^{\mathrm{H}_{2} \mathrm{O}}=459 \mathrm{meV}$ and $\nu(\mathrm{OH})_{\text {bound }}^{\mathrm{H}_{2} \mathrm{O}}=\nu_{0, \mathrm{ASW}}=423.6 \pm 0.2 \mathrm{meV}$. The density of such thicker water layers was here determined to be $0.59 \pm$ $0.16 \mathrm{~g} \mathrm{~cm}^{-3}$. The vanishing of the MUA substrate vibrational signature $\nu\left(\mathrm{CH}_{2}\right)$, caused by the masking effect of the deposited water layer, was used to estimate the depth probed by HREELS at $6 \mathrm{eV}$ to be $11 \pm 2 \AA$.

In this study, water molecules were used to probe the physical and chemical properties of a model hydrophilic organic organized layer. This method, relying on the HREELS analysis of samples exposed to water will be transposed in the near future to surfaces of various hydrophobicities. The water interaction with these substrates will then be characterized at the molecular level, and the structure of the water-interfacial layer will be studied.

\section{Acknowledgements}

We thank Dr Christophe Poulard for his help with the UV-Ozone treatment and Prof. Petra Swiderek for fruitful discussions. We acknowledge the financial support by C'Nano, Ile-de-France,
Programme Francilien de Recherche en Nanosciences, through the 2009 SAMTOX-NC granted project. This work was conducted within the framework of the COST action CM1301 (CELINA for 'Chemistry for ELectron-Induced NAnofabrication'). The HREELS electronics was funded by the LabEx PALM Investissement d'Avenir (ANR-10-LABX-0039-PALM, ProDac project).

\section{Notes and references}

1 M. A. Henderson, Surf. Sci. Rep., 2002, 46, 1-308.

2 P. A. Thiel and T. E. Madey, Surf. Sci. Rep., 1987, 7, 211-385.

3 F. Bensebaa and T. H. Ellis, Prog. Surf. Sci., 1995, 50, 173-185.

4 K. Jacobi, K. Bedürfig, Y. Wang and G. Ertl, Surf. Sci., 2001, 472, 9-20.

5 H. Ibach and S. Lehwald, Surf. Sci., 1980, 91, 187-197.

6 D. B. Asay and S. H. Kim, J. Phys. Chem. B, 2005, 109, 16760-16763.

7 C.-W. Yi and J. Szanyi, J. Phys. Chem. C, 2007, 111, 17597-17601.

8 W. Cantrell and G. E. Ewing, J. Phys. Chem. B, 2001, 105, 5434-5439.

9 I. Engquist, I. Lundström, B. Liedberg, A. N. Parikh and D. L. Allara, J. Chem. Phys., 1997, 106, 3038-3048.

10 I. Engquist, M. Lestelius and B. Liedberg, J. Phys. Chem., 1995, 99, 14198-14200.

11 R. G. Nuzzo, B. R. Zegarski, E. M. Koreniqt and L. H. Dubois, J. Phys. Chem., 1992, 96, 1355-1361.

12 L. H. Dubois, B. R. Zegarski and R. G. Nuzzo, J. Am. Chem. Soc., 1990, 112, 570-579.

13 L. Sun, R. M. Crooks and A. J. Ricco, Langmuir, 1993, 9, 1775-1780.

14 L. Sun, L. J. Kepley and R. M. Crooks, Langmuir, 1992, 8, 2101-2103.

15 F. Schreiber, J. Phys.: Condens. Matter, 2004, 16, R881.

16 A. Kudelski, Vib. Spectrosc., 2005, 39, 200-213.

17 N. Patel, M. C. Davies, M. Hartshorne, R. J. Heaton, C. J. Roberts, S. J. B. Tendler and P. M. Williams, Langmuir, 1997, 13, 6485-6490.

18 M. Wells, D. L. Dermody, H. C. Yang, T. Kim, R. M. Crooks and A. J. Ricco, Langmuir, 1996, 12, 1989-1996.

19 J. C. Love, L. A. Estroff, J. K. Kriebel, R. G. Nuzzo and G. M. Whitesides, Chem. Rev., 2005, 105, 1103-1170.

20 V. Humblot, P. Thebault, K. Boukerma, J.-F. Yala, A. Héquet, J.-M. Berjeaud and C.-M. Pradier, Biomaterials, 2009, 30, 3503-3512.

21 A. Vallée, V. Humblot, R. Al Housseiny, S. Boujday and C.-M. Pradier, Colloids Surf., B, 2013, 109, 136-142.

22 V. Molinero and B. D. Kay, J. Chem. Phys., 2014, 141, $18 \mathrm{C} 101$.

23 D. E. Brown, S. M. George, C. Huang, E. K. L. Wong, K. B. Rider, R. Scott Smith and B. D. Kay, J. Phys. Chem., 1996, 100, 4988-4995.

24 F. Tielens, D. Costa, V. Humblot and C.-M. Pradier, J. Phys. Chem. C, 2008, 112, 182-190.

25 G. A. Kimmel, K. P. Stevenson, Z. Dohnalek, R. Scott Smith and B. D. Kay, J. Chem. Phys., 2001, 114, 5284-5294. 
26 B. Guillot and Y. Guissani, J. Chem. Phys., 2004, 120, 4366-4382.

27 J. J. Pireaux, C. Gregoire and R. Caudano, Langmuir, 1991, 7, 2433-2437.

28 J. Houplin, L. Amiaud, V. Humblot, I. Martin, E. Matar, R. Azria, C.-M. Pradier and A. Lafosse, Phys. Chem. Chem. Phys., 2013, 15, 7220-7227.

29 R. Arnold, W. Azzam, A. Terfort and C. Wöll, Langmuir, 2002, 18, 3980-3992.

30 I. Pascal and D. S. Tarbell, J. Am. Chem. Soc., 1957, 79, 6015-6020.

31 R. V. Kasza, K. Griffiths, V. P. Zhdanov and P. R. Norton, Appl. Surf. Sci., 1995, 84, 97-105.

32 K. Jousten, Ultrahigh vacuum gauges CERN, 2007-24 p., CAS - CERN accelerator school and ALBA synchrotron light facility: course on vacuum in accelerators, Platja d'Aro, Spain, 16-24 May 2006.

33 K. Jousten, Pressure measurement with ionization gauges, OPEN-2000-271. - CERN, 1999 In: CAS - CERN accelerator school: vacuum technology, Snekersten, Denmark, 28 May-3 Jun 1999.

34 Varian Vacuum Technologies, Multi-gauge controller part no L8350301, 2000.

35 J. Houplin, PhD thesis, Université Paris Sud, 2015.

36 J. Houplin, L. Amiaud, T. Sedzik, C. Dablemont, D. TeilletBilly, N. Rougeau and A. Lafosse, Eur. Phys. J. D, 2015, 69, 217.

37 A. Lafosse and R. Azria, in Low-energy electron scattering from molecules, biomolecules and surfaces, ed. P. Čársky and R. Čurík, CRC Press Taylor \& Francis Group, 2012, ch. 7.
38 M. Allan, Phys. Rev. Lett., 2007, 98, 123201.

39 I. Martin, M. Bertin, A. Domanracka, R. Azria, E. Illenberger and A. Lafosse, Int. J. Mass Spectrom., 2008, 277, 262-268.

40 R. C. Millikan and K. S. Pitzer, J. Am. Chem. Soc., 1958, 80, 3515-3521.

41 National Institute of Standards and Technology (NIST), http://www.nist.gov.

42 S. E. Creager and C. M. Steiger, Langmuir, 1995, 11, 1852-1854.

43 H. C. Yang, D. L. Dermody, C. Xu, A. J. Ricco and R. M. Crooks, Langmuir, 1996, 12, 726-735.

44 R. G. Nuzzo, L. H. Dubois and D. L. Allara, J. Am. Chem. Soc., 1990, 112, 558-569.

45 J. J. Pireaux, C. Gregoire, M. Vermeersch, P. A. Thiry and R. Caudano, Surf. Sci., 1987, 189-190, 903-912.

46 P. Jenniskens, S. F. Banham, D. F. Blake and M. R. S. McCoustra, J. Chem. Phys., 1997, 107, 1232-1241.

47 G. G. A. Somorjai and M.-P. Delplancke, Chimie des surfaces et catalyse, Ediscience International, 1995.

48 K. Oura, V. G. Lifshits, A. A. Saranin, A. V. Zotov and M. Katayama, Surface science: an introduction, Springer, Berlin, 2003.

49 F. Schreiber, Prog. Surf. Sci., 2000, 65, 151-256.

50 Z. Dohnalek, G. A. Kimmel, P. Ayotte, R. Scott Smith and B. D. Kay, J. Chem. Phys., 2003, 118, 364-372.

51 Z. Dohnalek, R. L. Ciolli, G. A. Kimmel, K. P. Stevenson, R. Scott Smith and B. D. Kay, J. Chem. Phys., 1999, 110, 5489-5492.

52 B. D. Kay, K. R. Lykke, J. Randall Creighton and S. J. Ward, J. Chem. Phys., 1989, 91, 5120-5121. 